\title{
An FPGA-Based Upper-Limb Rehabilitation Device for Gesture Recognition and Motion Evaluation Using Multi-Task Recurrent Neural Networks
}

This paper was downloaded from TechRxiv (https://www.techrxiv.org).

\section{LICENSE}

CC BY 4.0

SUBMISSION DATE / POSTED DATE

$05-11-2021 / 11-11-2021$

\section{CITATION}

Liu, Haoyan; Panahi, Atiyehsadat; Andrews, David; Nelson, Alexander (2021): An FPGA-Based Upper-Limb Rehabilitation Device for Gesture Recognition and Motion Evaluation Using Multi-Task Recurrent Neural Networks. TechRxiv. Preprint. https://doi.org/10.36227/techrxiv.16943149.v1

$\mathrm{DOI}$ 


\title{
An FPGA-Based Upper-Limb Rehabilitation Device for Gesture Recognition and Motion Evaluation Using Multi-Task Recurrent Neural Networks
}

\author{
Haoyan Liu, Atiyehsadat Panahi, David Andrews, and Alexander Nelson
}

\begin{abstract}
Upper-Extremity motor impairment affects millions of Americans due to cerebrovascular incidents, spinal cord injuries, or brain trauma. Current therapy practices used to assist these individuals in regaining motor functionality often require extensive time at rehabilitation facilities with potentially prohibitive travel or financial costs. This work presents a mobile low-cost field programmable gate array (FPGA)-smart rehabilitation system that can be used in home environments. The prototype is a rehabilitation table instrumented with a capacitive sensor array (CSA) to track upper-extremity motions of the user through proximity or touch. In addition, inertial measurement units (IMUs) are placed on the affected upper limb and combined with the CSA data with our sensor

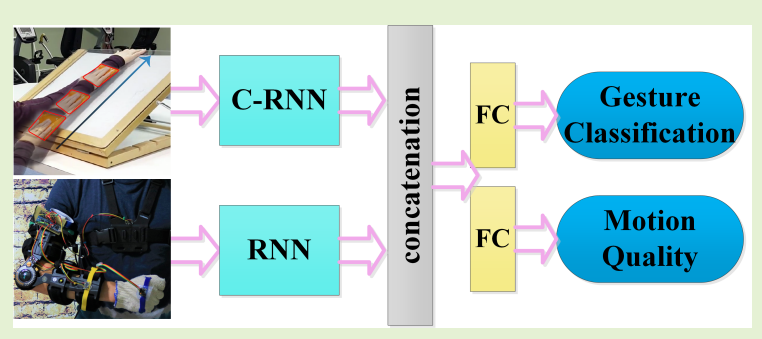
fusion signal processing architecture. Motions are classified and evaluated using multi-task convolutional recurrent neural networks with three additional motion quality output classes to personalize recognition based on the particular motor skills of each patient. The prototype achieves above $99 \%$ accuracy with 32-bit fixed-point format implementation for recognizing dynamic motions and identifying unnatural characteristics (i.e., tremor or limited flexion and extension) in upper limb motions based on sensor values. The convolutional recurrent neural network (C-RNN) fusion classification network is implemented on a $200 \mathrm{MHz}$ Zynq ZCU104 FPGA using an HLS-based design optimized with pipelining and parallelism techniques and achieves $5.4 \mathrm{x}$ speedup compared to ARM ${ }^{\circledR}$ Cortex-A53 implementation running at an operating frequency of $1.3 \mathrm{GHz}$. The prototype is also demonstrated to perform the machine learning classification in real-time.
\end{abstract}

Index Terms-Capacitive sensor array, deep learning, FPGA, gesture recognition, IMUs, neural networks, rehabilitation engineering

\section{INTRODUCTION}

C EREBROVASCULAR accident (CVA) is among the top five fatal causes in the U.S. [1]. Over 795, 000 American individuals are diagnosed with stroke each year, and above $76 \%$ of those individuals are newly diagnosed [1], [2]. Cerebrovascular accident costs the U.S. approximately $\$ 34 B$ annually, including healthcare services, prescriptions, and labor loss [1], [2]. Physical and occupational therapy sessions are common after CVA, spinal cord trauma, and brain injuries. While physical therapy is proven effective in improving motor functionalities and living quality [3], the expense of long-term treatment and the travel to outpatient rehabilitation centers can be major barriers to targeted longterm treatment [4]. This work develops a residential, smart, unobtrusive rehabilitation table with capacitive sensors, in-

The manuscript was submitted on November 4, 2021.

Haoyan Liu, Atiyehsadat Panahi, David Andrews, and Alexander Nelson are with the Department of Computer Science and Computer Engineering, University of Arkansas, Fayetteville, AR 72703 USA (email: \{hl002, apanahi, dandrews, ahnelson\}@uark.edu).

Haoyan Liu and Atiyehsadat Panahi contributed equally to this work. ertial measurement units (IMUs), and artificial neural network (ANN) classifiers for individuals with upper-extremity motor impairments due to CVA, spinal cord injuries, and/or brain trauma. Previous works have shown that the performance of ANN-based medical or recreational devices can be enhanced through personalization [5], [7]. The ability to reconfigure weights in classifiers through updating device firmware enables FPGAs to continually adapt to changing needs throughout a course of precision rehabilitation. Recent works have implemented ANNs on FPGAs due to their high performance, energy efficiency, and reconfigurability [8], [9]. In this work, the proposed personalized ANN-based sensor fusion classifiers are implemented using a pipelined HLSbased design and are evaluated in terms of performance, power, and resource utilization. The proposed custom ANNs are designed to provide high accuracy while utilizing fewer resources so the network parameters can be stored into only the on-chip FPGA memories.

The prototype is constructed from an off-the-shelf rehabilitation table, as shown in Fig. 1, with a capacitive sensor array (CSA) for upper-extremity motion trajectory sensing. 
Four 6-DoF IMUs (tri-axis accelerometer and gyroscope) are placed on the affected limb to provide a "ground truth" for kinematic parameters in addition to motion parameters such as tremor or compensatory motions from unaffected effectors (muscles). We adopt multi-task temporal neural network variants for gesture classification and motion quality evaluation. The IMUs provide accelerations and angular velocities in three dimensions at each joint along the affected upper limb. With a recurrent neural network, the IMU measurements are fused with capacitive sensor array readings to improve the classification accuracies of dynamic gesture and motion quality. One major benefit of using ANN models is that classification tasks can achieve high performance without engineering complex features. To validate the sensor fusion network architectures, we perform 5 -fold cross-validation with collected data.

The development of this prototype for upper-extremity motor rehabilitation provides the following contributions:

- A novel, real-time, RNN-based multi-task classification network that integrates sensor measurements from CSA and IMUs.

- Developing a deep learning fusion network to evaluate and classify the quality of motions from the user.

- Implementing an FPGA-based acceleration of the proposed classification network and evaluating its hardware performance and resource utilization when using parallelism and pipelining optimization techniques in different precisions and providing implementation trade-off insights.

The remainder of the paper is organized as follows: First, we present a review of existing literature and contrast their approach to our contributions. Second, we describe our novel classification network and its hardware implementation. Third, we evaluate the proposed contributions through a preliminary analysis, and discuss our assumptions and limitations. Finally, we conclude the paper and discuss future work.

\section{Related WORK}

\section{A. Sensors and Pattern Recognition}

Static and dynamic gesture recognitions are commonly used for device control and human activity recognition (HAR). A static gesture means keeping a certain pose without movement, whereas a dynamic gesture means a motion such as a swipe or a tap over sensors performed by users. Computer vision techniques are widely used to recognize gestures and human activities [10]-[13]. However, cameras can lead to privacy concerns for users, especially in residential environments, which limits their applications. Gesture recognition has become a common interaction pattern in recent years with

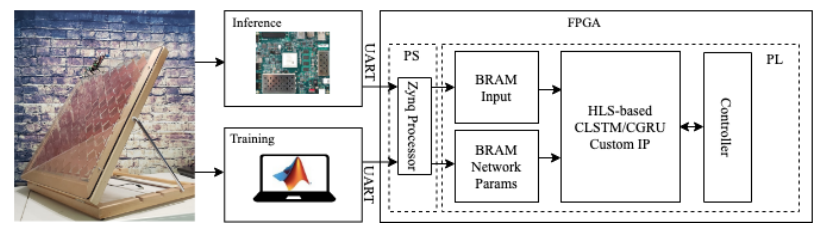

Fig. 1. An overview of the proposed system.
LiDAR, radar, infrared, resistive, and capacitive sensors [5][7], [14]-[18]. CapBand [7] is developed to recognize static hand gestures using capacitive sensors on a wrist band with a convolutional neural network (CNN). Tomo [14] is another wrist-worn device to classify hand gestures with electrical impedance tomography using a support vector machine classifier. Pyro [15] is a low-power, wearable thumb-tip dynamic gesture recognition device using a pyroelectric infrared sensing method based on a random forest classifier. We utilize and combine CSA and IMU readings with a neural network to classify gestures and motion quality performed by patients. For instance, a natural motion is free of trembling movements and joint stiffness for patients with upper limb extremity motor disabilities. This work also differs from [16], which utilizes a transmit-mode sensing method that requires skin contact on the capacitive sensor array, whereas our CSA can detect both proximity and touch. IMUs have been previously used for upper limb motion quality evaluation in [19]. The researchers engineered various features from IMU data and adopted a model tree classifier. Previous work [20] has proposed to use a multi-task recurrent network with an attention mechanism to monitor the multiple status of diagnoses. Our work uses a multi-task recurrent neural network based classifier that recognizes both dynamic gesture and motion quality with high accuracy.

\section{B. ANN Hardware Implementation}

CNNs are a common state-of-the-art backbone for ANNs [21]-[27]. This is because the convolution kernel enables training of fewer intermediate features through a biomimicry of the receptive field of the human visual cortex. Networks with fewer intermediate weights can enable deeper networks with similar training data and time. CNNs are typically used for static gesture recognitions, while recurrent neural network variants used for dynamic, time-sequential gesture classifications. By implementing gating structures within a memory cell for better gradient flow, long short-term memory (LSTM) neural network performs better than conventional recurrent neural network [28], [29]. Gated recurrent unit (GRU) networks with simplified structures have been introduced recently [30]. The performance comparison between GRU and LSTM neural networks is task-specific. In our work, we combine convolutional network and LSTM/GRU networks (C-LSTM/C-GRU) to extract spatial features per data frame from the capacitive sensor array using CNN and then apply temporal LSTM and GRU neural networks for classification.

FPGAs have been used to accelerate gesture recognition. FPGA accelerators can be categorized into two broad categories, namely 1) machine vision, and 2) ANN-based algorithms [10]-[13], [31]-[33]. Since the focus of this study is on the ANN-based algorithms for gesture recognition, the details of the machine vision algorithms are not discussed.

The Multilayer perceptron (MLP) is a basic feedforward ANN with activation functions, and has been accelerated in FPGA [34]. Another work combined MLPs with CNNs for FPGA image analysis [35]. More complex CNN networks are demonstrated in [36]-[38] for FPGA acceleration of gesture recognition algorithms. These methods implement hand 
motion recognition on a sequence of images captured by a camera. In these previous works, the input to the $\mathrm{CNN}$ network is a sequence of static images stored in DRAM, whereas, in our work, we perform a dynamic continuous gesture recognition on a touch-based capacitive sensor array. Similar to our work, [17] used the FPGA for sampling and basic digital signal processing algorithms, but not for classification. Moreover, they classified the gestures on a frequency-modulated continuouswave (FMCW) radar sensor, where our input is a multimodal sensor system of IMU and capacitive sensors. For these reasons, it is excluded in our comparisons. Other than performing classifications with CSA data, we fuse the CSA data with IMU measurements through an ANN to improve classification accuracy. We also modify basic recurrent neural networks to conduct multi-task classifications of dynamic gesture and gesture quality.

\section{Method And IMPLEMENTATION}

\section{A. System Overview}

The proposed system conducts motion recognition and quality analysis of rehabilitative exercises for patients with upper limb motor disabilities. As shown in Fig. 1, we use a capacitive sensor array to track dynamic gestures performed by patients. Furthermore, we use accelerations and angular velocities in three dimensions of the affected hand, forearm, upper arm, and upper body via IMUs to detect movement characteristics (i.e., tremor or joint stiffness) of an upper limb based on the dynamic gestures performed. In this work, motion quality is divided into three classes, which are natural motion, joint-constrained motion, and trembling motion. The number of classes can be easily extended based on patients' conditions. The results could be utilized to deduce the impairment severity level of patients and observe improvements of patients through the rehabilitation process. The four 6-DoF IMU sensors placed along the affected upper limb can be used to detect tremor, constrained, and normal motions, in addition to classification of gestures. The CSA can similarly identify these parameters. We propose to combine these sources of data as complementary measurements to obtain higher accuracy than either sensing modality can achieve in solidarity. Therefore, we fuse capacitive sensor data with IMU sensor values using recurrent neural network-based variants to respectively improve the classification performance of gesture pattern recognition and motion quality.

\section{B. Sensor Placement and Data Collection}

We use MSP430FR2676 MCU with specialized capacitive sensing analog front-end (AFE) using a charge-transfer technique [39] to obtain measurements of 64 capacitive sensors in an 8-by-8 configuration. The measurements at these sensors is given in charge transfer "counts" as the relative capacitance compared compared to the intrinsic and parasitic capacitance baseline of the circuitry. A structural ground layer is placed beneath the capacitive sensor array for shielding without largely decreasing the sensitivity. A customized 2layer PCB for capacitive sensing is shown in Fig. 2 (A). The capacitive sensors are built-in diamond shapes. We utilize mutual capacitance for this application. The upper limb under treatment is instrumented with four IMU sensors (MPU6050), which integrates an accelerometer and gyroscope. A subject wearing four IMUs is shown in Fig. 2 (B). The data of CSA and IMU sensors are logged on a PC via a UART connection at $250 \mathrm{kbps}$. The sampling rate of each sensor chip is set to 50 $\mathrm{Hz}$. The measurements of IMUs are resampled to match the measurements of the capacitive sensor array due to the clock differences of the two MCUs used to retrieve data.

IRB Protocol No. 2007274342 is obtained for evaluating the non-invasive sensor equipment with healthy human subjects. A collection of eight upper extremity motions are defined for rehabilitative training. The gestures are illustrated in Fig. 3. The data for training and testing are collected from 2 volunteers who are the paper authors. Both users have healthy full upper limb motor functionalities. Due to the COVID19 outbreak, we were unable to obtain IRB to perform tests on subjects in the intended target population with upper limb motor disabilities. To mimic possible complementary movements from the upper body and joint stiffness along with the upper limbs, we apply an arm brace to each user that can constrain the arm flexion and extension ranges. Each user performs every upper limb motion 30 times. The data collection contains three phases per user to eliminate the fatigue factor. During the first phase, the upper limb motions are collected with an arm brace with a full degree range of flexion and extension. In the second phase, upper limb motions are collected with an elbow brace with a constrained degree range of motions. Specifically, the extension and flexion are limited at $90^{\circ}$. During the third phase, we collect upper limb motion data injected with intended trembling characteristics. For each user, we collected 30 motion sequences per predefined gesture for each motion characteristic, resulting 1440 gesture sequences collected totally. We evaluate the ANN models with collected data in the following steps. First, the whole dataset is shuffled, and the gestures are split equally into 5 groups. Each group contains the same number of different gestures from different users. We then apply 5-fold leave-oneout cross-validation to test a variety of network models with different hyperparameters such as hidden units in recurrent neural network cells and the number of recurrent network layers. The ratio of training and test dataset is $4: 1$. Finally, we obtain high-performance ANN models for further investigation and hardware implementation.

\section{ANN-Based Classifier Models}

To assist the rehabilitation process with patients experiencing upper-extremity motor disabilities, the system needs to know whether a patient successfully performed an upper limb motion that is consistent with a pre-defined rehabilitative gesture database. The system also needs to identify whether the upper limb motion is performed naturally and close to the ones performed by individuals with healthy upper limb motor functions. We explore a variety of ANN models utilizing CSA and IMU sensor values respectively or jointly to accurately discriminate upper limb motions among our aforementioned gesture database. The classification accuracy 
of network models is compared between using sole features obtained from CSA, sole features from IMUs, and joined measurement features from CSA and IMUs. Specifically, we first try to use two recurrent neural networks to conduct dynamic gesture recognition and gesture quality classification in parallel, using measurements either from CSA or IMUs. One of our hypothesizes is that the CSA placed beneath the activity table can recognize gesture patterns well but cannot precisely identify the quality of a gesture, such as how the gesture is performed with the upper limb. To address the gesture quality classification problem of upper limb motions, we use IMU readings at the forearm, upper arm, and upper body as inputs to ANN-based models. These locations were chosen to obtain joint angles and motions through acceleration and angular velocity. For classification using only CSA data, we apply recurrent neural network variants such as LSTM, GRU, convolutional LSTM (C-LSTM), and convolutional GRU (CGRU) networks. We add convolutional operations to extract spatial features since 8-by-8 capacitive sensor data at each time frame is akin to a low-resolution image. For classification using IMU measurements alone, we apply basic LSTM and GRU neural networks. Then, we fuse sensor measurements from both CSA and IMUs with a deep learning network and pass the data into two parallel multiple-input, singleoutput (MISO) recurrent networks as shown on the left side of Fig. 4. The parallel MISO recurrent networks can be trained separately. However, each recurrent neural network with 88 input features is duplicated to achieve both classification tasks. At last, we pass the joined measurements into our proposed multiple-input, multiple-output (MIMO) fusion network structure shown on the right side of Fig. 4 and trained in a custom loop using MATLAB ${ }^{\circledR}$. The training loss function consists of two cross-entropy terms [20] respectively for gesture recognition and gesture quality classification is given as in (1). The discounting factor $\lambda_{g}$ and $\lambda_{q}$ are set to be equal. $P_{o g, c g}$ represents the predicted probability observation $o g$ is of class $c g . P_{o q, c q}$ represents the predicted probability observation $o q$ is of class $c q . M$ and $N$ are the number of gesture classes and the number of gesture quality classes, respectively.

$$
y=-\lambda_{g} \sum_{c g=1}^{M} y_{o g, c g} \log P_{o g, c g}-\lambda_{q} \sum_{c q=1}^{N} y_{o q, c q} \log P_{o q, c q}
$$

Prior to the implementation on FPGA for further analysis, we simulated both 16-bit and 32-bit fixed-point ANN implementations in MATLAB. Utilizing fixed-point weight implementations instead of floating-point implementations leads to less hardware resource usage and faster computation. These simulations guided the implementation of the FPGA classifiers.

\section{Hardware Implementations of ANN Classifiers}

The inference phase for the personalized classification networks is implemented in C++ on the HLS tool. The synthesized hardware description language (HDL) is extracted as a custom IP to be implemented on the FPGA. The system overview of the design is shown in Fig. 1. As presented in

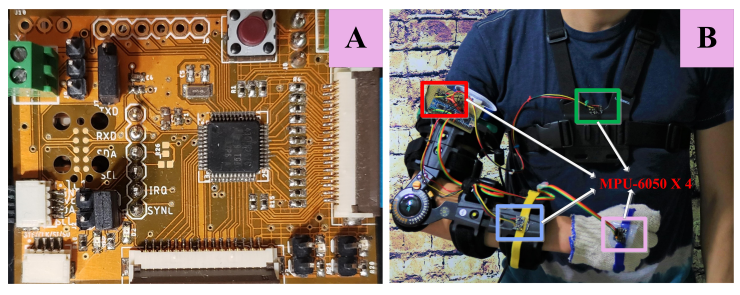

Fig. 2. (A) A customized capacitive sensor board; (B) a subject wearing 4 IMUs along the upper limb.

this figure, the custom IP is used along with a block RAM (BRAM) for storing input data. The input data is a sequence of data frames. Each data frame contains 64 measurements representing the capacitive sensors and 24 measurements from the IMUs. We pass those data to the custom IP frame by frame. The controller is connected to the IP and keeps track of input data frames (in discrete time windows) using handshake signals with the IP. In the inference phase, the input data is collected from the capacitive and IMU sensors and passed to the FPGA fabric using a UART connection. The trained network parameters are stored in the BRAMs, which are accessible to the IP for fast data transfer. The custom IP is a C-CLSTM/C-GRU which classifies the input sequences into 8 gesture classes represented in Fig. 3 and three separate classes of motion quality, which are natural motion, constrained joint motion, and trembling motion. The reason for using recurrent neural networks over conventional CNNs is implementing dynamic gesture recognition. The input data consists of a sequence of data in different time windows sampled every 20 ms $(50 \mathrm{~Hz})$. The motion is segmented through a threshold of positive capacitive sensing activity while the user is conducting the gesture on the capacitive sensor array table.

The network parameters and arithmetic operations are implemented in both fixed-point and floating-point data formats. The fixed-point design results in the same accuracy with faster and smaller hardware. In the fixed-point designs, the overflow is handled using the wrap-around method and the fixed-point values are quantized to the closest representable number in the direction of negative infinity. The Sigmoid activation function is developed using the PLAN method [40] that archives lower resource utilization and execution time compared to [41], [42] and can be implemented using only shift and addition operations that are easily implemented in hardware. The Tanh function is also implemented using the PLAN-based Sigmoid function since as proposed in [43] and presented in (2),

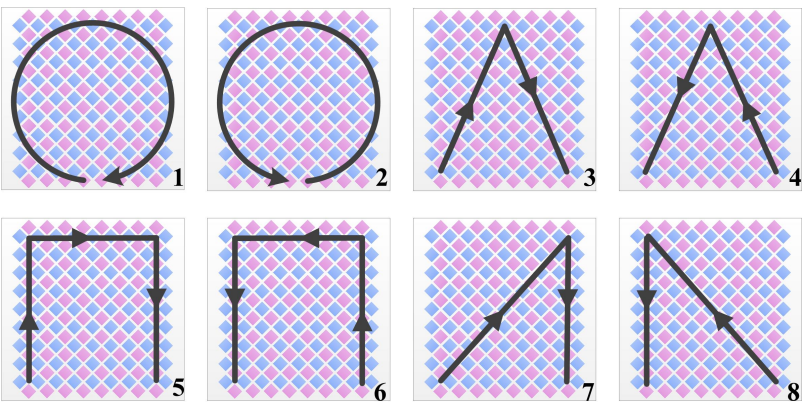

Fig. 3. A collection of upper limb motions. 


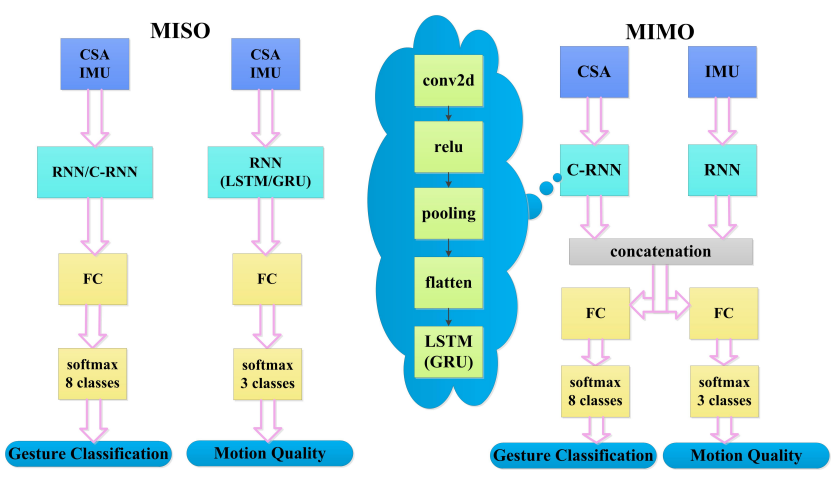

Fig. 4. Single-output v.s. multiple-output network structures.

$\operatorname{Tanh}(x)$ can be computed based on $\sigma(-2 x)$.

$$
\operatorname{Tanh}(x)=1-2 \times \sigma(-2 x)
$$

Matrix-vector multiplication (MVM), as the main operation of LSTM and GRU networks, is also optimized using the column-based method originally proposed in [44]. We have pipelined the MVM operations to achieve a higher operating frequency and improve performance. In addition, we implemented loop-unrolling and evaluated its performance in section IV.

\section{Results ANd Discussion}

\section{A. Sensor Measurements}

The capacitive sensor MSP430FR2676 is configured to scan the entire CSA at $50 \mathrm{~Hz}$ with four channels in parallel. There are a total of 64 channels for the 8-by-8 low-resolution CSA. These 64 measurements for each sensor in the CSA are the input features of our ANN models. Each MPU6050 measures accelerations and angular velocities in three dimensions, resulting in 6-dimensional tuple per sensor. There are four MPU6050s placed along the arm wearing an arm brace. Thus, there are a total of 24 variables obtained from IMUs each time step. Instead of a traditional signal processing pipeline for the inertial sensors, we send the full resolution data into the neural networks to combine features between IMUs and capacitive sensors using a data-driven methodology.

The sensor values from CSA and IMUs are recorded and segmented when the CSA is being touched using an adaptive threshold. A 2-dimensional time-sequential dynamic clockwise circle gesture captured by CSA from each user are shown in Fig. 5. For clarity, each image frame of CSA is resized to $224 \times 224$ pixels using "bicubic" interpolation. The white pixels indicate the hand location with respect to the CSA. A sequence sample of measurements obtained from IMUs, when each user was performing a clockwise circle gesture, is shown in Fig. 6. The distributions of the sequence length of each user are respectively shown in Fig. 7. From the data comparison between subjects, it is found that subjects perform the same gestures differently and personalized rehabilitation devices may be preferable. For instance, user 1 generally performs gestures faster than user 2. This is in agreement with prior work [5]. In this work, we train our network with data from both subjects.

\section{B. ANN-based Classification}

The results of comparing ANN models is summarized in Table I. We adopt $8 \times 10^{-4}$ as the learning rate for network training by balancing the training speed and performance. We use a batch size of 4 for training. To better visualize gesture and motion quality classification accuracies of various models with and without feature fusion, results in Table I are presented in Fig. 8. For single-input, single-output (SISO) networks, as shown in Fig. 8, with the CSA data alone, the recurrent neural network variants can differentiate dynamic gestures with high accuracy but are unable to recognize the quality of a gesture accurately. With IMU data alone, the ANN models can recognize the quality of a gesture better than classify the
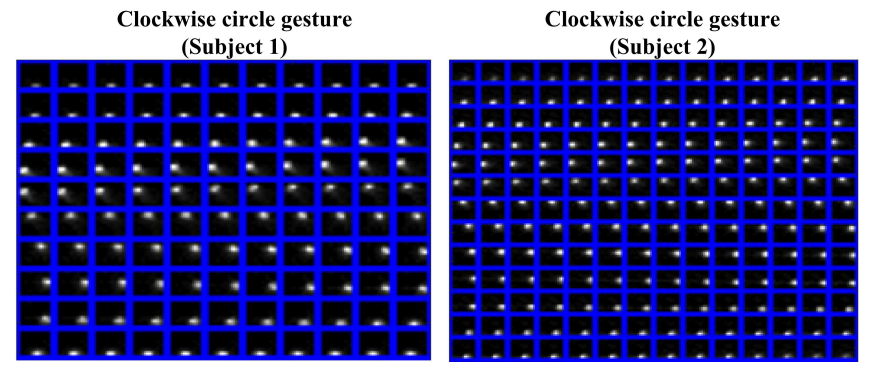

Fig. 5. A comparison of 2-dimentional, time-sequential CSA measurements of the clockwise circle gesture performed by different subjects.
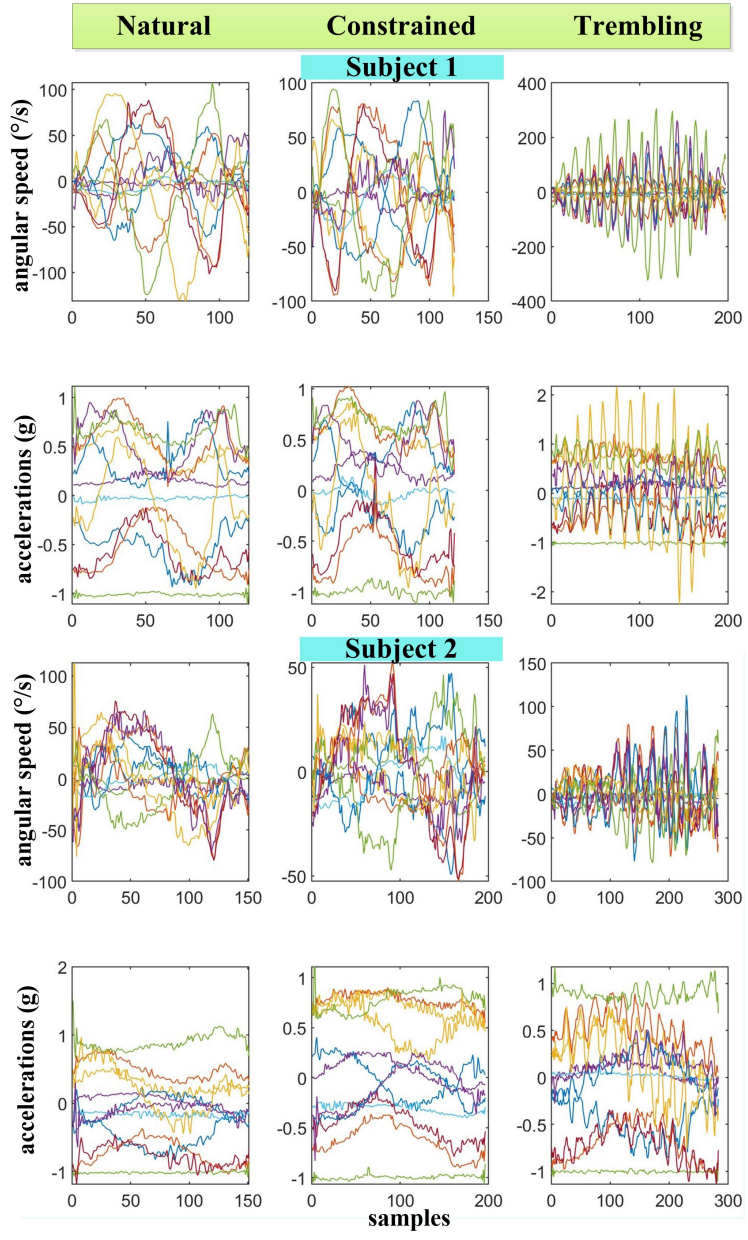

Fig. 6. Tri-axis acceleration and angular velocity measurements from IMUs when the clockwise circle gesture is performed differently by subjects. 


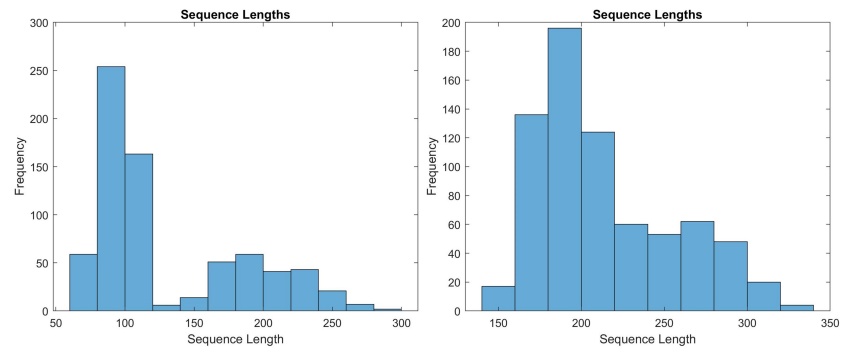

Fig. 7. The distributions of gesture length collected from each subject.

dynamic gesture. The highest gesture classification accuracy achieved among the tested SISO models is $99.88 \%$ using a CGRU model. The C-GRU model uses four $3 \times 3$ kernels without any paddings in the convolution layer. The stride size is 1 . In the down-sampling pooling layer, the window and stride size are both $2 \times 2$. For IMU-alone gesture quality classification, a two-layer LSTM network with 64 hidden nodes in each layer outperformed similar models in our testing. Among the MISO models with both features from CSA and IMUs, we observe that the 2-layer GRU model with 64 hidden nodes in each layer outperforms other models when averaging the accuracies of gesture recognition and quality classification. Among the LSTM-based MIMO fusion networks, the best training results are from Model 31 with 16 kernels, two LSTM layers of 64 hidden nodes for the CSA data, and one LSTM layer of 64 hidden nodes for IMU measurements. However, the numbers of parameters and operations are higher than other models. Among all the MIMO fusion networks, Model 34 with 16 kernels, one GRU layer of 64 hidden nodes for the CSA data, and one GRU layer of 64 hidden nodes for IMU data achieves the best classification accuracy for both gesture recognition and motion quality evaluation. It is found that MIMO models with an average pooling layer perform slightly better than similar models with a max-pooling layer. The proposed MIMO sensor fusion network structure outperforms parallel MISO models (e.g. Model 12 for gesture recognition and Model 23 for motion quality classification) while using fewer parameters. The total parameters separated by "/" and listed in each row of Table I respectively represent network parameters for gesture recognition and motion quality classification task. The confusion matrices of the 5-fold crossvalidation results for both gesture recognition and quality classification are shown in Fig. 9. The diagonal blocks in green show the classification accuracy of each class, whereas the percentages in the far-right column of each confusion matrix show the classification precision of each class.

\section{Fixed-Point Simulation}

Before deploying our ANN models on FPGA, we conduct simulations in MATLAB using 16-bit and 32-bit fixed-point format parameters and operations to observe any diminutions in prediction accuracy. We use the PLAN method for state and gate activation functions in the inference phase. Floor rounding and wrap-around on overflow are utilized. We use 16-bit fraction length for 32-bit fixed-point and 8-bit fraction length for 16-bit fixed-point. We use this same floating-point structure in the FPGA implementation. The results are shown in Table II. Here we simulate and compare an LSTM variant network with a GRU variant model, which achieves the best classification performance as shown in Table I, using 64 hidden nodes in each recurrent layer. When the parameters are implemented using the 16-bit fixed-point format, the gesture recognition accuracy drops around 3\% using Model 27 with 16-bit fixed-point format parameters, whereas the gesture recognition accuracy reduces less than $0.1 \%$ using Model 34 . The classification accuracy of motion quality drops less than $2.5 \%$ for both models, respectively. With 32-bit fixed-point format implementation, the classification accuracy remains the same as floating-point implementation.

\section{FPGA Implementation}

The proposed gesture recognition MIMO networks are accelerated on a Zynq-ZCU104 Ultrascale+ FPGA using Vivado (2018.3). The performance, power, and resource utilization in terms of look-up tables (LUTs), flip-flops (FFs), BRAMs, and digital signal processing (DSPs) of post place-and-route results are presented for both C-LSTM and C-GRU networks in Table III. For this table, we implemented the network with the best simulated results found from the experiment shown in Table I (i.e. MIMO Model 27 and 34). In the reported results of this table, all designs are pipelined for matrix-multiplication and parallelized in gates of LSTM and GRU cells. Loop-unrolling is utilized in the CNN layer over the number of channels.

The effects of row-based and column-based methods for matrix-vector multiplication operations are evaluated for both fixed-point and floating-point data formats. Table III is the results of comparison between row-based and column-based methods. The table shows the utilized resources are decreased in the column-based method by a factor of up to $4.3 x, 2.7 x$, and $1.6 x$ in total usage of LUTs and FFs, BRAMs, and DSPs compared to their equivalent row-based designs. These resource savings come without a significant increase in execution time. The results for C-GRU networks follow the same pattern when comparing the row-based and column-based methods. In general, as GRU networks are simplified over LSTMs, the execution-time, resource utilization, and power consumption is decreased in C-GRU networks compared to equal C-LSTMs. It is worth mentioning that in all the network designs in Table III, from the total available BRAM and ultra RAM (URAM) on-chip memories of the ZCU-104 FPGA, only BRAM is utilized and the URAM utilization is zero. The highlighted networks in this table require $0.2 \mathrm{MB}$ memory that is around $14.5 \%$ of the total available $1.375 \mathrm{MB}$ of the on-chip BRAM. The 3.375 MB on-chip URAM memory of this FPGA is left unused. Thus, the proposed design can also be implemented on a cost-effective FPGA.

As expected, the designs with floating-point data formats result in higher execution time, resource, and BRAM utilization compared to their equivalent fixed-point designs in 32 and 16 bits data-widths. Table III does show some cases where lower LUTs utilization in floating-point designs results in higher FFs utilization, and therefore a higher utilized resource percentage. Comparing the DSP utilization in these two methods shows 
TABLE I

FIVE-FOLD VALIDATION RESULTS OF VARIOUS NEURAL NETWORK

\begin{tabular}{|c|c|c|c|c|c|}
\hline ID & & Total & Gesture & Quality & $\mathrm{CNN}$ \\
\hline & Layer & Params & Accuracy & Accuracy & Layer \\
\hline \multicolumn{6}{|c|}{ SISO models: CSA data only (64 channels) } \\
\hline 1 & LSTM (32) & $12680 / 12515$ & $99.40 \%$ & $82.42 \%$ & - \\
\hline & LSTM (64) & $33544 / 33219$ & $99.68 \%$ & $87.08 \%$ & \\
\hline & LSTM $(32,32)$ & $21000 / 20835$ & $99.68 \%$ & $80.02 \%$ & - \\
\hline 4 & LSTM $(64,64)$ & $66568 / 66243$ & $99.48 \%$ & $83.40 \%$ & \\
\hline & C-LSTM (64) & $26416 / 26091$ & $97.94 \%$ & $67.70 \%$ & {$[3,3] \times 4, \operatorname{maxPool}$} \\
\hline 6 & C-LSTM $(64,64)$ & $59440 / 59115$ & $98.56 \%$ & $66.12 \%$ & {$[3,3] \mathrm{X} 4, \max$ Pool } \\
\hline & GRU (32) & $9576 / 9411$ & $99.24 \%$ & $82.16 \%$ & \\
\hline & GRU (64) & $25288 / 24963$ & $99.74 \%$ & $84.02 \%$ & \\
\hline & GRU $(32,32)$ & $15816 / 15651$ & $99.66 \%$ & $79.32 \%$ & - \\
\hline$\frac{10}{11}$ & $\begin{array}{l}\text { GRU (64, 64) } \\
\text { C-GRU (64) }\end{array}$ & $\frac{50056 / 49731}{19952 / 19627}$ & $\frac{99.66 \%}{99.62 \%}$ & $85.28 \%$ & $\frac{-}{[3,3] \times 4, \operatorname{maxPool}}$ \\
\hline 12 & C-GRU $(64,64)$ & $44720 / 44395$ & $99.88 \%$ & $74.74 \%$ & {$[3,3] \times 4$, maxPool } \\
\hline \multicolumn{6}{|c|}{\begin{tabular}{|l} 
SISO models: IMU data only (24 channels) \\
\end{tabular}} \\
\hline$\frac{13}{14}$ & $\frac{\operatorname{LSTM}(32)}{\text { LSTM (64) }}$ & $7560 / 7395$ & & $83.32 \%$ & - \\
\hline 15 & $\begin{array}{l}\operatorname{LSTM}(64) \\
\operatorname{LSTM}(128)\end{array}$ & $\frac{23304 / 22979}{79368 / 78723}$ & $74.38 \%$ \% & $\frac{84.16 \%}{84.52 \%}$ & - \\
\hline & $\operatorname{LSTM}(32,32)$ & $15880 / 15715$ & $74.64 \%$ & $87.24 \%$ & \\
\hline 17 & LSTM $(64,64)$ & $56328 / 56003$ & $78.42 \%$ & $88.56 \%$ & - \\
\hline \multirow{2}{*}{\multicolumn{6}{|c|}{$\begin{array}{l}\text { MISO models: CSA+IMU data (88 channels) } \\
\end{array}$}} \\
\hline 18 & LSTM (32) & & & $99.32 \%$ & \\
\hline & $\begin{array}{ll}\text { LSTM (64) } \\
\end{array}$ & $39688 / 39363$ & $96.12 \%$ & $99.18 \%$ & \\
\hline 20 & LSTM $(32,32)$ & $24072 / 23907$ & $91.20 \%$ & $98.28 \%$ & \\
\hline & LSTM $(64,64)$ & $72712 / 72387$ & $99.12 \%$ & $93.82 \%$ & 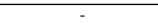 \\
\hline & GRU (32) & $11880 / 11715$ & $90.42 \%$ & $97.02 \%$ & \\
\hline & GRU (64) & $29896 / 29571$ & $94.10 \%$ & $99.68 \%$ & \\
\hline 24 & GRU $(32,32)$ & $18120 / 17955$ & $92.98 \%$ & $98.56 \%$ & \\
\hline 25 & GRU $(64,64)$ & $54664 / 54339$ & $98.84 \%$ & $99.58 \%$ & \\
\hline \multicolumn{6}{|c|}{$\begin{aligned} \text { MIMO models: CSA+IMU data (88 channels) } \\
\end{aligned}$} \\
\hline 26 & C-LSTM(32), LSTM(32) & & & $99.20 \%$ & {$[3,3]$ X $\quad 16$, avgPool } \\
\hline & C-LSTM(64), LSTM(64) & 77867 & $99.60 \%$ & $99.60 \%$ & {$[3,3] \mathrm{X} 16$, avgPool } \\
\hline & C-LSTM(32, 32), LSTM(32) & 39147 & $98.70 \%$ & $99.04 \%$ & {$[3,3] \mathrm{X} \quad 16$, avgPool } \\
\hline & C-LSTM(64, 64), LSTM( & 83123 & $95.14 \%$ & $99.18 \%$ & {$[3,3]$ X 4, avgPool } \\
\hline 30 & C-LSTM(64, 64), LSTM( 6 & 110891 & $99.72 \%$ & $99.68 \%$ & {$[3,3]$ X 16, maxPool } \\
\hline & (644, 64), LSTM(64) & 110891 & $99.88 \%$ & $99.74 \%$ & {$[3,3] \mathrm{X} \quad 16$, avgPool } \\
\hline 32 & (32), GRU & 23339 & $99.88 \%$ & $99.40 \%$ & {$[3,3] \times 16$, avgPool } \\
\hline 33 & & 587 & 99.9 & $99.72 \%$ & IX $16, \mathrm{~m}$ \\
\hline 34 & & 587 & 99.9 & 99.74\% & {$[3,3]$ X 16, avgPool } \\
\hline 35 & $C-G$ & & 99.74\% & 99.24\% & {$[3,3]$ X 16, avgPool } \\
\hline 36 & C-GRU(64, 64), GRU(64) & & $99.68 \%$ & $99.58 \%$ & {$[3,3]$ X 4, avgPool } \\
\hline 37 & C-GRU(64, 64), GRU(64) & 83563 & $99.74 \%$ & $99.68 \%$ & {$[3,3] \mathrm{X} 16$, avgPool } \\
\hline
\end{tabular}

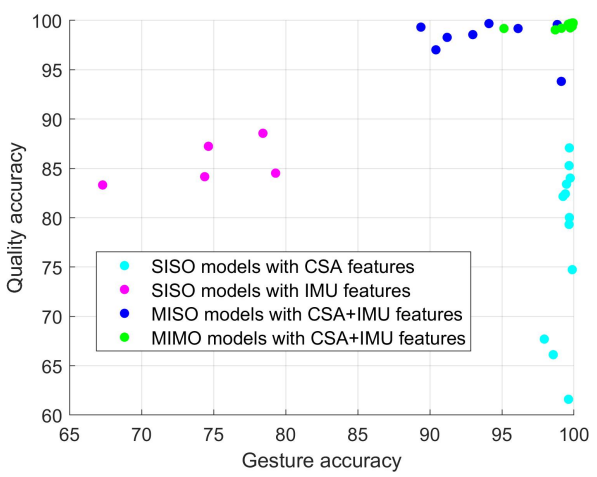

Fig. 8. Classification accuracy of various models with different input features.

TABLE II

FIXED-POINT ANALYSIS OF ANN MODELS

FIXED-POINT ANALYSIS OF ANN MODELS
\begin{tabular}{|c|c|c|c|c|}
\hline & \multicolumn{2}{|c|}{ 16-bit Fixed Point } & \multicolumn{2}{c|}{ 32-bit Fixed Point } \\
\hline & Gesture Acc. & Quality Acc. & Gesture Acc. & Quality Acc. \\
\hline C-LSTM(64), LSTM(64) & $96.12 \%$ & $97.29 \%$ & $99.60 \%$ & $99.60 \%$ \\
\hline C-GRU(64), GRU(64) & $99.86 \%$ & $97.29 \%$ & $99.94 \%$ & $99.74 \%$ \\
\hline
\end{tabular}

a lower number of DSPs in floating-point compared to fixedpoint. In the designs with the same 32 bits for fixed-point and floating-point data formats, the lower DSP utilization is reflected in higher LUTs, FFs, and BRAMs' utilization in the floating-point methods. The DSP blocks are designed for fixedpoint operations and so in the fixed-point operations, the DSPs are preferred over other resources for arithmetic operations, while in the floating-point operations, the built-in floatingpoint IP in the Vivado is utilized for the arithmetic operations. Comparing the fixed-point results shows that as expected, for lower data-widths, the power and resource utilization is decreased and the performance is improved. However, based on Table II, the accuracy of the 16-bit design does not change significantly when compared to the 32-bit design. The power presented in III is the average of reported power in the Vivado tool and the Xilinx Power Estimator (XPE). Power consumption in this table has a direct relation with the utilized resources and DSPs of each design. As previously discussed, the power consumption of C-GRU networks is lower than the equivalent C-LSTM network and the row-based methods also consume more power than the equal columnbased methods. Comparing the power consumption of floatingpoint and fixed-point methods show that in some cases, the power consumption is higher in the fixed-point method, which is the result of higher DSP utilization in this method so that around $30 \%$ of the dynamic power consumption results from DSPs.

In addition to pipelining which is applied to all methods in Table III, one design in both C-LSTM and C-GRU networks includes loop-unrolling optimization over the number of channels in the CNN layer. As the results show, the resource utilization is significantly higher than the equivalent method, while the execution time is not improved significantly. Since as presented in Table I, II, the accuracy of the fixed-point and floating-point data formats is in the same order and also considering the performance, resource utilization, and power consumption of different presented methods, the optimum method is bolded in Table III. In this method, fixed-point data type and column-based matrix-vector multiplication are utilized and it provides an optimum point between resource utilization, performance, and power consumption. As we have utilized our custom-designed fusion networks for the gesture classification (using CSA) as well as identifying abnormal characteristics (using IMU), we could not compare the FPGA implementation results with the current machine learning accelerators in the literature, since our network designs are not the same as them.

The execution time of the FPGA-based implemented networks are compared to $\mathrm{ARM}^{\circledR}$ Cortex-A53 implementation in Table IV. As the ARM processor supports floating-point operations, we have compared the floating-point FPGA implementation of our proposed networks while using the same column-based MVM method for both ARM and FPGA PL implementation. The ARM processor runs at its maximum frequency of $1.3 \mathrm{GHz}$ and the FPGA fabric is running at 200 MHz. The results show the lower execution time of the FPGA fabric implementation, which is mainly because of higher memory access time in the processor.

\section{LIMITATIONS}

This work has the following limitations: First, due to the COVID-19 outbreak and reducing human contacts as precautions, we have not been able to collect upper limb motion data from the target user group, who are often in long-term care facilities and belong to a high-risk group. This work has been validated by limited data collected from the authors who have full upper limb motor functions. As such, this work is focused on the hardware acceleration and combination of IMU and CSA data for technological feasibility. The accuracy on the initial two subjects was sufficient to demonstrate 

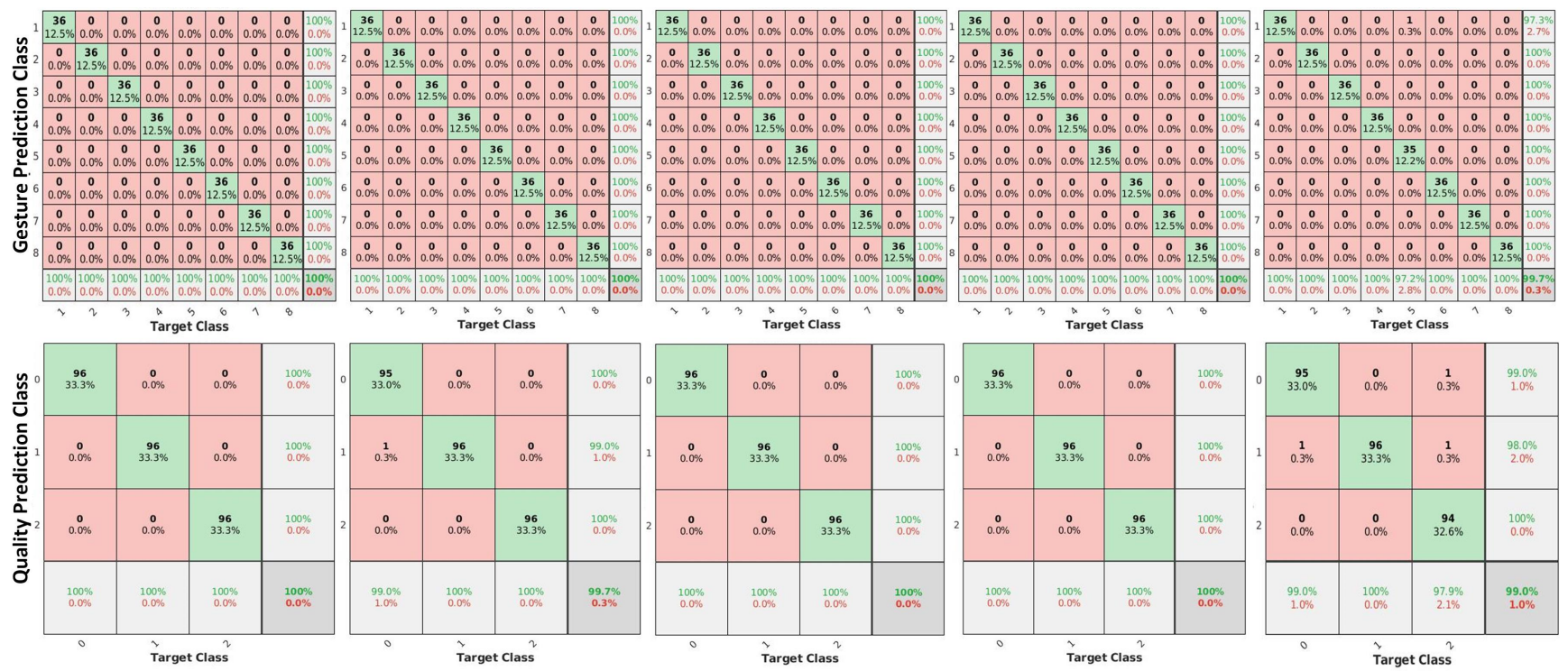

Fig. 9. Confusion matrices of the classification results of the MIMO neural network (top: gesture recognition; bottom: quality classification).

TABLE III

MIMO FPGA IMPLEMENTATION RESULTS

\begin{tabular}{|c|c|c|c|c|c|c|c|c|}
\hline $\begin{array}{l}\text { Data } \\
\text { Format }\end{array}$ & $\begin{array}{l}\text { Exe-time } \\
(\mathrm{ms})\end{array}$ & $\begin{array}{c}\text { Frequency } \\
\text { (MHz) }\end{array}$ & $\begin{array}{l}\text { MVM } \\
\text { method }\end{array}$ & LUTs & FFs & BRAMs $^{1}$ & DSPs & $\begin{array}{l}\text { Avg Power } \\
\text { (W) }\end{array}$ \\
\hline \multicolumn{9}{|c|}{$\begin{array}{l}\text { MIMO C-LSTM(64), LSTM(64) } \\
\end{array}$} \\
\hline FlP 32-bit & 0.68 & 200 & Row & 43576 & 64259 & 299 & 125 & 1.86 \\
\hline FxP 32-bit & 0.30 & 200 & Row & 30633 & 25434 & 120 & 892 & 2.36 \\
\hline FxP 16-bit & 0.28 & 200 & Row & 8146 & 6179 & 86 & 560 & 1.70 \\
\hline FIP 32-bit & 0.81 & 200 & Col & 11019 & 13794 & 109 & 95 & 1.10 \\
\hline FxP 32-bit & 0.44 & 200 & $\mathrm{Col}$ & 13886 & 9258 & 61 & 538 & 1.68 \\
\hline FxP 32-bit & 0.43 & 200 & $\mathrm{Col}$ & 19434 & 14343 & 69 & 568 & $1.90^{2}$ \\
\hline FxP 16-bit & $\mathbf{0 . 4 2}$ & 200 & Col & 5393 & 5046 & 36 & 140 & 0.96 \\
\hline \multicolumn{9}{|c|}{ MIMO C-GRU(64), GRU(64) } \\
\hline FlP 32-bit & 0.63 & 200 & Row & 32406 & 57941 & 292 & 122 & 1.63 \\
\hline FxP 32-bit & 0.27 & 200 & Row & 20601 & 28094 & 104 & 888 & 1.64 \\
\hline FxP 16-bit & 0.24 & 200 & Row & 7472 & 5579 & 83 & 559 & 1.64 \\
\hline FlP 32-bit & 0.73 & 200 & $\mathrm{Col}$ & 10266 & 13311 & 74 & 92 & 1.04 \\
\hline FxP 32-bit & 0.37 & 200 & Col & 13427 & 9150 & 54 & 534 & 1.59 \\
\hline FxP 32-bit & 0.36 & 200 & Col & 18675 & 14301 & 61 & 564 & $1.83^{2}$ \\
\hline FxP 16-bit & 0.34 & 200 & Col & 5047 & 4930 & 32 & 139 & 0.95 \\
\hline \multicolumn{9}{|c|}{ Total Available Resources } \\
\hline \multicolumn{4}{|c|}{ N/A } & 230,400 & 460,800 & 312 & 1728 & N/A \\
\hline
\end{tabular}

${ }^{1}$ URAM utilization in all designs is zero.

${ }^{2}$ In addition to pipelined MVM, loop-unrolling optimization is also applied to the CNN part.

TABLE IV

EXECUTION TIME COMPARISON

\begin{tabular}{|c|c|c|c|c|c|}
\hline & \multicolumn{2}{|c|}{ PL (CPGA Fabric) } & PS (Zynq ARM Processor) & Improvement \\
\hline & Exe (ms) & Freq (MHz) & Exe (m) & Freq (GHz) & (x) \\
\hline MIMO (C-LSTM) & 0.81 & 200 & 4.45 & 1.3 & 5.4 \\
\hline MIMO (C-GRU) & 0.73 & 200 & 3.52 & 1.3 & 4.8 \\
\hline
\end{tabular}

feasibility, and to obtain system specifications. To demonstrate sufficiency for the target population, an additional follow-up study will need to be performed when Covid-19 isolation protocols subside. Additionally, the accuracy in two different task domains presented in this work demonstrates the success of deep learning-based sensor fusion technique without the need of complex data pre-processing and feature engineering. As previously mentioned, we have only evaluated the athome rehabilitation device with subjects who have full, healthy motor functions. To mimic the movement characteristics that people with upper extremity motor disabilities may present, the subjects wore an arm brace that constrains the extension and flexion range of the right-sided elbow. The brace is shown in Fig. 2(B), and the dials in that figure are adjustable to prevent extension beyond those angles. Trembling movements were simulated by the users when performing gestures that can be discriminated against by the designed neural network. These tremors are not representative of actual tremor but were collected to determine whether abnormal movements may be identified compared to the baseline. We understand that the motor-skills of people from people with upper extremity motor impairments will be heterogenous. The model however is sufficient to identify the types of networks and classification techniques that will be used in a deployed system. The neural computation hardware platform is reconfigurable and can be easily adapted to new deep learning models and/or parameters 
for the targeted user group or new classification sets.

\section{CONCLUSION AND FUtURE WORK}

This paper develops and evaluates an FPGA-based timeseries gesture recognition system with CSA and IMUs that can be used for constructing a smart, at-home upper-extremity motor impairment system. The proposed approach is capable of distinguishing upper limb motion characteristics during rehabilitation when interacting with the rehabilitation table using a multi-task RNN network. In this work, we implemented a C-LSTM/C-GRU based MIMO network on a Zynq FPGA using HLS code optimized with pipelining, parallelism, and loop-unrolling techniques. We also compare row-based and column-based MVM methods for fixed-point and floatingpoint data formats. The proposed network for gesture classification achieves $5.4 x$ speedup compared to ARM $^{\circledR}$ Cortex-A53 platform implementation.

In the future, we will conduct a study over more subjects, especially with patients who have upper extremity motor impairments. We will implement a user-friendly interface to provide real-time classification feedback to users. In addition, we will integrate the entire system from sensing to processing in the FPGA. Additionally, we will explore the use of multitask RNN to predict the motion quality in an early stage of a gesture.

\section{REFERENCES}

[1] E. J. Benjamin, M. J. Blaha, S. E. Chiuve, M. Cushman, S. R. Das, R Deo, et al., "Heart disease and stroke statistics-2017 update: a report from the American Heart Association," Circulation, vol. 135, no. 10, 2017.

[2] Stroke Facts, Centers for Disease Control and Prevention, January 31, 2020. [Online]. Available:https://www.cdc.gov/stroke/facts.htm

[3] C. J. Winstein, D. K. Rose, S. M. Tan, R. Lewthwaite, H. C. Chui, and S. P. Azen, "A randomized controlled comparison of upper-extremity rehabilitation strategies in acute stroke: a pilot study of immediate and long-term outcomes," Archives of Physical Medicine and Rehabilitation, vol. 85, no. 4, pp. 620-628, Apr. 2014.

[4] K. M. Godwin, J. Wasserman, and S. K. Ostwald, "Cost associated with stroke: outpatient rehabilitative services and medication," Top Stroke Rehabil., vol. 18, pp. 676-684, 2011.

[5] A. Nelson, S. M. Waller, R. Robucci, C. Patel, and N. Banerjee, "Evaluating touchless capacitive gesture recognition as an assistive device for upper extremity mobility impairment," Journal of Rehabilitation and Assistive Technologies Engineering, vol. 5, pp. 1--13, 2018.

[6] G. Cohn, S. Gupta, T. Lee, D. Morris, J. R. Smith, M. S. Reynolds, D. S. Tan, and S. N. Patel, "An ultra-low Power human body motion sensor using static electric field sensing," In the Proceedings of ACM Ubicomp 2012, pp. 99-102, 2012.

[7] H. Truong, S. Zhang, U. Muncuk, P. Nguyen, N. Bui, A. Nguyen, et al., "CapBand: battery-free successive capacitance sensing wristband for hand gesture recognition,' In Proceedings of the 16th ACM Conference on Embedded Networked Sensor Systems, pp. 54-67, 2018.

[8] A. Yazdanbakhsh, M. Brzozowski, B. Khaleghi, S. Ghodrati, K. Samadi, N. S. Kim, et al., "FlexiGAN: An end-to-end solution for FPGA acceleration of generative adversarial networks," 2018 IEEE 26th Annual International Symposium on Field-Programmable Custom Computing Machines (FCCM), Boulder, CO, pp. 65-72, 2018.

[9] L. Lu, J. Xie, R. Huang, J. Zhang, W. Lin and Y. Liang, "An efficient hardware accelerator for sparse convolutional neural networks on FPGAs," 2019 IEEE 27th Annual International Symposium on FieldProgrammable Custom Computing Machines (FCCM), San Diego, CA, USA, pp. 17-25, 2019.

[10] T.H. Tsai, Y. C. Ho, and Y.R. Tsai, "Implementation of 3D Hand Gesture Recognition System using FPGA," In 2018 International SoC Design Conference (ISOCC), pp. 131-132, 2018.
[11] M. Raj, I. Deepan, M. Gogul, V. Thangaraja, and K. Sathiesh, "Static gesture recognition based precise positioning of 5-DOF robotic arm using FPGA," In 2017 Trends in Industrial Measurement and Automation (TIMA), pp. 1-6, 2017.

[12] C. T. Li, and W. Chen, "A novel FPGA-based hand gesture recognition system," Journal of Convergence Information Technology, vol. 7, no. 9, pp. 221-229, 2012.

[13] F.J. Toledo-Moreo, J.J. Martinez-Alvarez, and J.M. Ferrandez-Vicente, "Hand-based interface for augmented reality," In 15th Annual IEEE Symposium on Field-Programmable Custom Computing Machines (FCCM), pp. 291-292, 2007.

[14] Y. Zhang and C. Harrison, "Tomo: Wearable, low-cost electrical impedance tomography for hand gesture recognition," In Proceedings of the 28th Annual ACM Symposium on User Interface Software and Technology, pp. 167-173, 2015.

[15] J. Gong, Y. Zhang, X. Zhou, and X. Yang, "Pyro: Thumb-Tip gesture recognition using pyroelectric infrared sensing," In Proceedings of the 30th Annual ACM Symposium on User Interface Software and Technology, pp. 553-563, 2017.

[16] H. Liu, A. Panahi, D. Andrews, and A. Nelson, "FPGA-Based gesture recognition with capacitive sensor array using recurrent neural networks," 2020 IEEE 28th Annual International Symposium on FieldProgrammable Custom Computing Machines (FCCM), Fayetteville, AR, USA, pp. 225-225, 2020.

[17] Z. Zhang, T. Zengshan, and Z. Mu, "Latern: Dynamic continuous hand gesture recognition using FMCW radar sensor," IEEE Sensors Journal, no. 8, pp. 3278-3289, 2018.

[18] v. V. Menon, S. A. Siddiqui, S. Rao, A. Schmidt, M. French, V. Chirayath, and A. Li, "Design and Performance Evaluation of Multispectral Sensing Algorithms on CPU, GPU, and FPGA," In 2021 IEEE Aerospace Conference (50100), pp. 1-9, 2021.

[19] H. Jung, J. Park, J. Jeong, T. Ryu, Y. Kim, and S. I. Lee, "A wearable monitoring system for at-home stroke rehabilitation exercises: A preliminary study," 2018 IEEE EMBS International Conference on Biomedical \& Health Informatics (BHI), Las Vegas, NV, 2018, pp. 13 16.

[20] Q. Suo, F. Ma, G. Canino, J. Gao, A. Zhang, P. Veltri, and A. Gnasso, "A multi-task framework for monitoring health conditions via attentionbased recurrent neural networks," AMIA Аnпи Symp Proc., pp. 16651674, 2018.

[21] J. Faraone, M. Kumm, M. Hardieck, P. Zipf, X. Liu, D. Boland, and P. H. Leong, "AddNet: Deep Neural Networks Using FPGA-Optimized Multipliers," IEEE Transactions on Very Large Scale Integration (VLSI) Systems, vol. 28, no. 1, pp. 115-128, 2019.

[22] W. Zhao, H. Fu, W. Luk, T. Yu, S. Wang, B. Feng, et al., "F-CNN: An FPGA-based framework for training convolutional neural networks," In 2016 IEEE 27th International Conference on Application-specific Systems, Architectures and Processors (ASAP), pp. 107-114, 2016.

[23] M. Sit, R. Kazami, and H. Amano, "FPGA-based accelerator for losslessly quantized convolutional neural networks," In 2017 International Conference on Field Programmable Technology (ICFPT), pp. 295-298. 2017.

[24] R. Zhao, X. Niu, Y. Wu, W. Luk, and Q. Liu, "Optimizing CNNbased object detection algorithms on embedded FPGA platforms," In International Symposium on Applied Reconfigurable Computing, pp. 255-267, 2017

[25] N. J. Fraser, Y. Umuroglu, G. Gambardella, M. Blott, P. Leong, M. Jahre, and K. Vissers, "Scaling binarized neural networks on reconfigurable logic," In Proceedings of the 8th Workshop and 6th Workshop on Parallel Programming and Run-Time Management Techniques for Manycore Architectures and Design Tools and Architectures for Multicore Embedded Computing Platforms, pp. 25-30, 2017.

[26] G. Zhong, A. Dubey, C. Tan, and T. Mitra, "Synergy: An hw/sw framework for high throughput cnns on embedded heterogeneous soc," ACM Transactions on Embedded Computing Systems (TECS), vol. 18, no. 2, pp. 1-23, 2019.

[27] R. Zhao, H. C. Ng, W. Luk, and X. Niu, "Towards efficient convolutional neural network for domain-specific applications on FPGA," In 2018 28th International Conference on Field Programmable Logic and Applications (FPL), pp. 147-1477, 2018.

[28] Z. Que, Y. Liu, C. Guo, X. Niu, Y. Zhu, W. Luk, "Real-Time anomaly detection for flight testing using autoEncoder and LSTM," In 2019 International Conference on Field-Programmable Technology (ICFPT), pp. 379-382, 2019.

[29] S. Wang, Z. Li, C. Ding, B. Yuan, Q. Qiu, Y. Wang, and Y. Liang, "C-LSTM: Enabling efficient LSTM using structured compression techniques on FPGAs," In Proceedings of the 2018 ACM/SIGDA Inter- 
national Symposium on Field-Programmable Gate Arrays, pp. 11-20, 2018.

[30] E. Nurvitadhi, J. Sim, D. Sheffield, A. Mishra, S. Krishnan, and D. Marr, "Accelerating recurrent neural networks in analytics servers: Comparison of FPGA, CPU, GPU, and ASIC," In 2016 26th International Conference on Field Programmable Logic and Applications (FPL), pp. 1-4, 2016.

[31] R. Tapiador-Morales, J. M. Maro, A. Jimenez-Fernandez, G. JimenezMoreno, R. Benosman, and A. Linares-Barranco, "Event-Based Gesture Recognition through a Hierarchy of Time-Surfaces for FPGA," Sensors, vol. 20, no. 12, pp. 3404, 2020.

[32] U. T. Salim, S. A. Dawwd, "Systolic hand gesture recognition/detection system based on FPGA with multi-port BRAMs," Alexandria Engineering Journal, vol. 58, no. 3, pp. 841-848, 2019.

[33] W. Song, Q. Han, Z. Lin, N. Yan, D. Luo, Y. Liao, et al., "Design of a flexible wearable smart sEMG recorder integrated gradient boosting decision tree based hand gesture recognition ," IEEE Transactions on Biomedical Circuits and Systems, vol. 13, no. 6, pp. 1563-1574, 2019.

[34] V. T. Huynh, "Design of artificial neural network architecture for handwritten digit recognition on FPGA," 2016.

[35] L. B. Saldanha, and C. Bobda, "Sparsely connected neural networks in FPGA for handwritten digit recognition," In 2016 17th International Symposium on Quality Electronic Design (ISQED), pp. 113-117, 2016.

[36] T. Zhang, Z. Weiguo, X. Jiang, and Y. Liu, "FPGA-based Implementation of Hand Gesture Recognition Using Convolutional Neural Network," In 2018 IEEE International Conference on Cyborg and Bionic Systems (CBS), pp. 133-138, 2018.

[37] R. Núñez-Prieto, G. Pablo Correa, and L. Liang, "A Real-Time Gesture Recognition System with FPGA Accelerated ZynqNet Classification," In 2019 IEEE Nordic Circuits and Systems Conference (NORCAS): NORCHIP and International Symposium of System-on-Chip (SoC), pp. $1-6,2019$.

[38] H. Fan, H.C. Ng, S. Liu, Z. Que, X. Niu, and W. Luk, "Reconfigurable Acceleration of 3D-CNNs for Human Action Recognition with Block Floating-Point Representation," In 28th International Conference on Field Programmable Logic and Applications (FPL), pp. 287-2877, 2018.

[39] Texas Instruments, "MSP430FR267x Capacitive Touch Sensing MixedSignal Microcontrollers ," MSP430FR267x datasheet, Mar. 2019 [Revised Feb. 2020].

[40] H. Amin, K.M. Curtis, and B.R. Hayes-Gill, "Piecewise linear approximation applied to nonlinear function of a neural network," IEEE Proceedings-Circuits, Devices and Systems, vol. 144, no. 6, pp.313-317, 1997.

[41] O. Cetin, F. Temurtas, and S. Gulgonul, "An application of multilayer neural network on hepatitis disease diagnosis using approximations of sigmoid activation function," Dicle Medical Journal/Dicle Tip Dergisi, vol. 42, no. 2, 2015.

[42] Chad Greene (2020). sigmoid (https://www.mathworks.com/matlabcentral/fileexchange/51007sigmoid), MATLAB Central File Exchange. Retrieved January 29, 2020.

[43] S. Gomar, M. Mirhassani, and M. Ahmadi, "Precise digital implementations of hyperbolic tanh and sigmoid function," In 50th Asilomar Conference on Signals, Systems and Computers, pp. 1586-1589, 2016.

[44] Z. Que, H. Nakahara, E. Nurvitadhi, H. Fan, C. Zeng, J. Meng, X. Niu, and W. Luk, "Optimizing reconfigurable recurrent neural networks," In 2020 IEEE 28th Annual International Symposium on FieldProgrammable Custom Computing Machines (FCCM), pp. 10-18, 2020. 